\title{
Consecutive mutations leading to the emergence in vivo of imipenem resistance in a clinical strain of Enterobacter aerogenes
}

\author{
L. S. TZOUVELEKIS, E. TZELEPI, M. E. KAUFMANN* and A. F. MENTIS
}

Department of Bacteriology, Hellenic Pasteur Institute, Vas. Sofias 127, Athens 11521, Greece and

* Gram-negative Unit, Laboratory of Hospital Infection, PHLS, 61 Colindale Avenue, London NW9 $5 H T$

\begin{abstract}
Summary. Three consecutive isolates of Enterobacter aerogenes were obtained from the blood cultures of a hospitalised patient who was receiving antibiotic therapy. The initial isolate possessed an inducible cephalosporinase and was susceptible to third-generation cephalosporins. After ceftazidime treatment, a second isolate resistant to this antibiotic and characterised by stable overproduction of the chromosomal $\beta$-lactamase was obtained, and therapy was altered to a new combination which included imipenem. During this course of treatment, a strain of $E$. aerogenes was isolated that was resistant to virtually all $\beta$-lactam agents including imipenem. Comparison of biotypes and ribotyping profiles indicated that the three isolates were probably derived from a single strain which had undergone several mutations during antibiotic exposure. Examination of outer-membrane protein (OMP) preparations and lipopolysaccharide (LPS) profiles showed that the imipenem-resistant isolate lacked a major OMP and high molecular mass LPS. Furthermore, this isolate displayed reduced permeability to cephaloridine compared with the initial isolate. The introduction of a plasmid carrying a wild-type $\operatorname{ampD}$ allele prevented cephalosporinase production and restored $\beta$-lactam susceptibility in the imipenem-resistant isolate. It was concluded that stable derepression of class-I $\beta$-lactamase production and reduced permeability are both required for expression of imipenem resistance in $E$. aerogenes, and that previous exposure to cephalosporins may encourage the emergence of such strains.
\end{abstract}

\section{Introduction}

Enterobacter aerogenes is often associated with hospital-acquired infections, particularly in immunocompromised patients. In common with other members of the genus Enterobacter, exposure to thirdgeneration cephalosporins results in a high frequency of emergence of $\beta$-lactam-resistant mutants that produce large amounts of chromosomal cephalosporinase constitutively. ${ }^{1}$ Imipenem is considered to be stable to the hydrolytic activity of chromosomal cephalosporinase, and thus should retain its efficacy. ${ }^{2}$ However, isolation of imipenem-resistant variants of $E$. aerogenes, selected either in vitro or in vivo, has been reported. ${ }^{3-6}$ In this report, we describe the development in vivo of imipenem resistance in a strain of $E$. aerogenes after combined therapy, and suggest that emergence of imipenem resistance may be enhanced by previous exposure to cephalosporins.

\section{Materials and methods}

\section{Case report}

A 67-year-old leukaemic patient was admitted to hospital with high fever $\left(39^{\circ} \mathrm{C}\right)$ and low blood pressure. Before the onset of empirical treatment with a combination of ceftazidime $(1 \mathrm{~g}$ i.v. $/ 8 \mathrm{~h})$ plus netilmicin (150 $\mathrm{mg}$ i.v. $/ 8 \mathrm{~h}$ ), blood, sputum and urine samples were cultured. From the blood culture, a strain of $E$. aerogenes (isolate $\mathrm{E} 1$ ), susceptible to the above drugs, was isolated. After initial improvement, the patient's temperature increased to $39.5^{\circ} \mathrm{C}$ on day 13 . Again, $E$. aerogenes (isolate E2) was isolated from blood cultures. This isolate was resistant to virtually all $\beta$ lactam agents except imipenem. The antibiotic regimen was changed to imipenem/cilastatin ( 1 g i.v. $/ 8 \mathrm{~h}$ ) plus ciprofloxacin $(0.2 \mathrm{~g}$ i.v. $/ 12 \mathrm{~h})$. From one of the subsequent blood cultures (day 19), a slowly growing $E$. aerogenes strain (isolate E3) was obtained. This isolate was resistant to all $\beta$-lactam agents, including imipenem, and also displayed reduced susceptibility to fluoroquinolones, as evaluated by standard antibiograms. The patient was treated with sulphamethoxazole/trimethoprim for 5 more days. On day 
32 after admission the patient died from multiple system organ failure.

\section{Bacterial strains}

Isolates were identified as $E$. aerogenes with the API20E system (bioMérieux, La Balme Les Grottes, France). Further biotyping was performed by studying sugar utilisation patterns with the API50CH system (bioMérieux).

\section{Transformation experiments}

Caesium chloride-purified DNA of plasmid pNH5 (comprising a $\mathrm{HpaI}$ fragment containing the $a m p D^{+}$ gene from Escherichia coli, cloned into pBS18) ${ }^{7}$ was used to transform the clinical isolates. Transformants were selected on MacConkey agar (Oxoid) containing kanamycin (Sigma) $64 \mathrm{mg} / \mathrm{L}$. Plasmid pNH5 was kindly provided by $\mathrm{S}$. T. Cole, Institut Pasteur, Paris, France. The preparation of $E$. aerogenes competent cells and the transformation procedure were as described previously. ${ }^{8}$

\section{Ribotyping}

Ribotyping was performed as described previously. ${ }^{9}$ Briefly, chromosomal DNA was digested with EcoRI (New England BioLabs, Beverly, MA, USA), subjected to agarose gel electrophoresis and transferred to nylon membranes (Hybond-N; Amersham). Fragments containing rDNA genes were localised by hybridisation with a cDNA probe transcribed by reverse transcriptase (New England BioLabs) from $E$. coli rRNA (Boehringer-Mannheim). The probe was labelled with biotin-16-dUTP (BoehringerMannheim) by the random primer method. ${ }^{10}$ Fragments showing positive hybridisation with the labelled probe were detected with a streptavidin-alkaline phosphatase conjugate (BluGENE kit; Gibco-BRL).

\section{Antibiotic susceptibility}

Susceptibility testing was performed in MuellerHinton (MH) broth (Oxoid) with the microdilution technique recommended by the National Committee for Clinical Laboratory Standards (NCCLS). ${ }^{11}$ Antibiotics were purchased from commercial sources.

\section{$\beta$-Lactamase studies}

Preparation of crude $\beta$-lactamase extracts and cefoxitin induction experiments were performed as described previously. ${ }^{12}$ Protein content was measured with a Protein Assay Kit (BioRad). Cephaloridine hydrolysis rates were determined by UV spectrophotometry at a wavelength of $295 \mathrm{~nm}$, and were expressed as units of hydrolytic activity ( 1 unit = $1 \mathrm{nmol}$ of antibiotic hydrolysed $/ \mathrm{min} / \mathrm{mg}$ of protein). Analytical iso-electric focusing (IEF) was performed on polyacrylamide gels as described previously, ${ }^{13}$ with an ampholyte of $\mathrm{pH} \mathrm{3 \cdot 5-9.5} \mathrm{(Pharmacia).} \beta$ Lactamase bands were visualised with nitrocefin (Oxoid); pI values were determined by comparison with standard $\beta$-lactamases of known $\mathrm{pI}$ values.

\section{Characterisation of outer-membrane proteins (OMPs)}

OMP preparations were derived from mid-logphase cultures in $\mathrm{MH}$ broth by ultrasonic treatment in a MSE sonicator $(15 \mu \mathrm{m}$ for $2 \mathrm{~min})$, followed by differential solubilisation ${ }^{14}$ of the cytoplasmic material with sodium lauryl sarcosinate (Sigma). The final preparations were electrophoresed on polyacrylamide (acrylamide $12 \% \mathrm{w} / \mathrm{v}$ ) gels containing SDS and stained with Coomassie Brilliant Blue.

\section{Lipopolysaccharide (LPS) profiles}

LPS profiles were determined after silver staining of whole cell preparations treated with proteinase $K$ and run on urea-polyacrylamide gels. ${ }^{15}$

\section{Assessment of cephaloridine permeation rates}

Cephaloridine permeability coefficients, expressed as nmol of antibiotic hydrolysed/sec, were determined by comparing the rate of antibiotic hydrolysis by intact and sonicated cells as described previously. ${ }^{16}$ Hydrolytic rates for intact cells were corrected for enzyme leakage by subtracting the $\beta$-lactamase activities of the supernates of the cell suspensions used.

\section{Results}

All three isolates of $E$. aerogenes had the same biochemical profile with the API20E (profile number 5305773) and API50CH systems. Together with the similarity of ribotyping patterns (fig. 1), this fact suggests that the isolates were derived from the same original strain.

The initial isolate (E1) was susceptible to thirdgeneration cephalosporins and imipenem. The production of $\beta$-lactamase by this isolate increased at least 50 -fold after induction with cefoxitin (table). The second isolate (E2) was resistant to the cephalosporins tested, but the MIC of imipenem was elevated only slightly. This isolate was a constitutive overproducer of $\beta$-lactamase. The imipenem-resistant isolate (E3) also produced, constitutively, large amounts of $\beta$ lactamase. The cephalosporin MICs for isolate E3 were higher than those for isolate E2 (table). All three isolates possessed a single $\beta$-lactamase of pI $8 \cdot 2$ (fig. 2).

Attempts to transform isolates E2 and E3 with the amp $D^{+}$-encoding plasmid pNH5 were successful only with isolate E3. Examination of one of the transformants (E3T4) showed that low, non-inducible levels of $\beta$-lactamase were produced. Concomitantly, susceptibility to imipenem was restored, but MICs of 


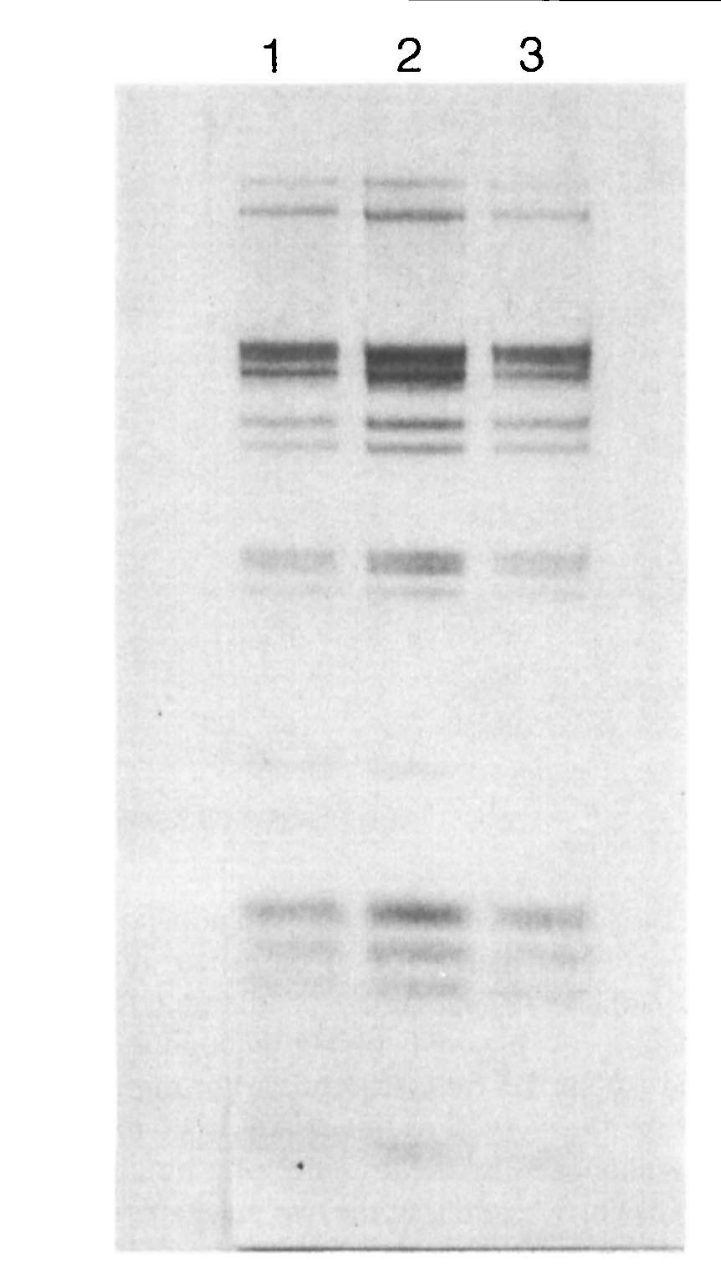

Fig. 1. Ribotyping patterns of the three $E$. aerogenes clinical isolates produced following digestion of chromosomal DNA with EcoRI Lane 1 , isolate $\mathrm{E} 1 ; 2$, isolate $\mathrm{E} 2 ; \mathbf{3}$, isolate $\mathrm{E} 3$.

Table. MICs, $\beta$-lactamase activities and permeability coefficients for the $E$. aerogenes clinical isolates (E1, E2 and E3) and an $a m p D^{+}$transformant (E3T4)

\begin{tabular}{|c|c|c|c|c|c|c|}
\hline \multirow{2}{*}{ Isolate } & \multicolumn{3}{|c|}{$\mathrm{MIC}(\mathrm{mg} / \mathrm{L})$} & \multicolumn{2}{|c|}{$\beta$-Lactamase activity } & \multirow{2}{*}{$\begin{array}{l}\text { Permeability } \\
\text { coefficient* }\end{array}$} \\
\hline & CAZ & CTX & IPM & Uninduced & Induced & \\
\hline E1 & 2 & 2 & 0.5 & 58 & 2961 & 248 \\
\hline $\mathrm{E} 2$ & 128 & 256 & 1 & 3516 & 3310 & 231 \\
\hline E3 & $>256$ & $>256$ & 16 & 2855 & 2950 & 31 \\
\hline E3T4 & 4 & 8 & 0.5 & $<20$ & $<20$ & $\ldots$ \\
\hline
\end{tabular}

CAZ, ceftazidime; CTX, cefotaxime; IPM, imipenem.

* Expressed as nmol of cephaloridine hydrolysed/s. Each coefficient represents the mean value of five calculations (each of which differed individually from the mean by $<10 \%$ ).

cephalosporins for E3T4 were slightly higher than those for isolate E1.

The OMP profiles of isolates E1 and E2 were identical, but isolate E3 differed in lacking, almost totally, the $39-\mathrm{kDa}$ major protein (fig. 3A). Furthermore, the imipenem-resistant isolate (E3) appeared not to express high molecular mass $\mathrm{O}$-antigenic LPS side chains (fig. 3B).

Evaluation of the outer-membrane permeability of the three isolates indicated that isolate E3 was substantially less permeable to cephaloridine than isolates

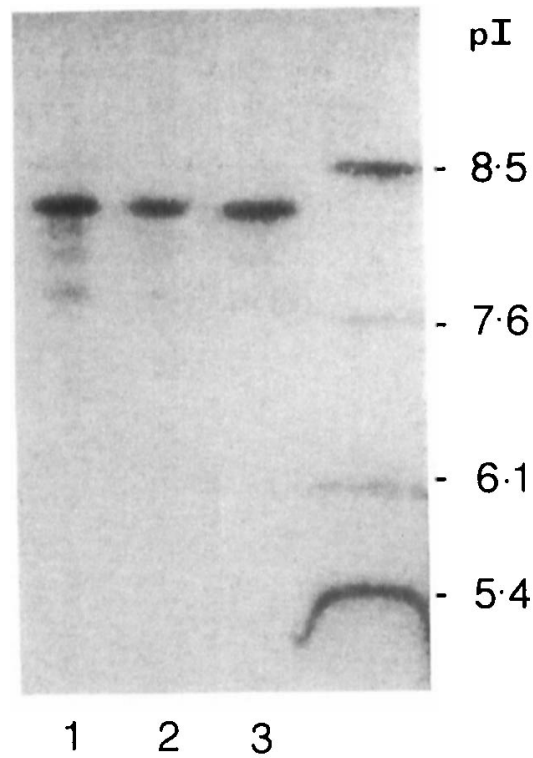

Fig. 2. IEF of crude enzyme preparations from the three $E$. aerogenes clinical isolates. Lane 1 , isolate $\mathrm{E} 1 ; 2$, isolate $\mathrm{E} 2 ; 3$, isolate $\mathrm{E} 3$. Also shown on the right are $\beta$-lactamases of known pI value (TEM-1, pI 5.4; PSE-2, pI 6.1; SHV-1, pI 7.6; and AmpC from E. cloacae strain $\mathrm{MHN} 1$, pI 8.5)

E1 and E2. However, the methodology followed was insensitive and 5-10\% differences were observed in repeated experiments.

\section{Discussion}

Imipenem-resistant clinical isolates of Pseudomonas aeruginosa and Acinetobacter spp. emerge, at relatively high frequencies, soon after the introduction of imipenem in the clinical setting. Detailed studies with $P$. aeruginosa have shown that the main mutational event leading to resistance is the loss of an imipenem-specific porin termed D2. ${ }^{17,18}$ In enterobacteria, it seems that imipenem does not prefer any specific porin channel and this might explain the fact that most of the imipenem-resistant enterobacterial isolates described to date have displayed reduced expression of the main porin $\mathrm{F}$.

Although loss of this major porin species has been correlated with the emergence of imipenem-resistant strains of Enterobacter, quantitative models suggest that impaired permeability alone cannot explain highlevel imipenem resistance. ${ }^{19,20}$ Derepressed overproduction of the class-I chromosomal $\beta$-lactamase has been suggested as the additional synergic requirement for the expression of resistance. This mechanism has been well documented in imipenem-resistant strains of E. cloacae. ${ }^{21,22}$ Recently, DeChamps and co-workers reported that imipenem-resistant $E$. aerogenes strains isolated in French hospitals were characterised by overproduction of class-I $\beta$-lactamase and loss of a major OMP, ${ }^{6}$ and in a study of resistant mutants of $E$. aerogenes selected in vitro by imipenem, we obtained evidence that loss of porin(s) and cephalosporinase derepression were both required for expression of 


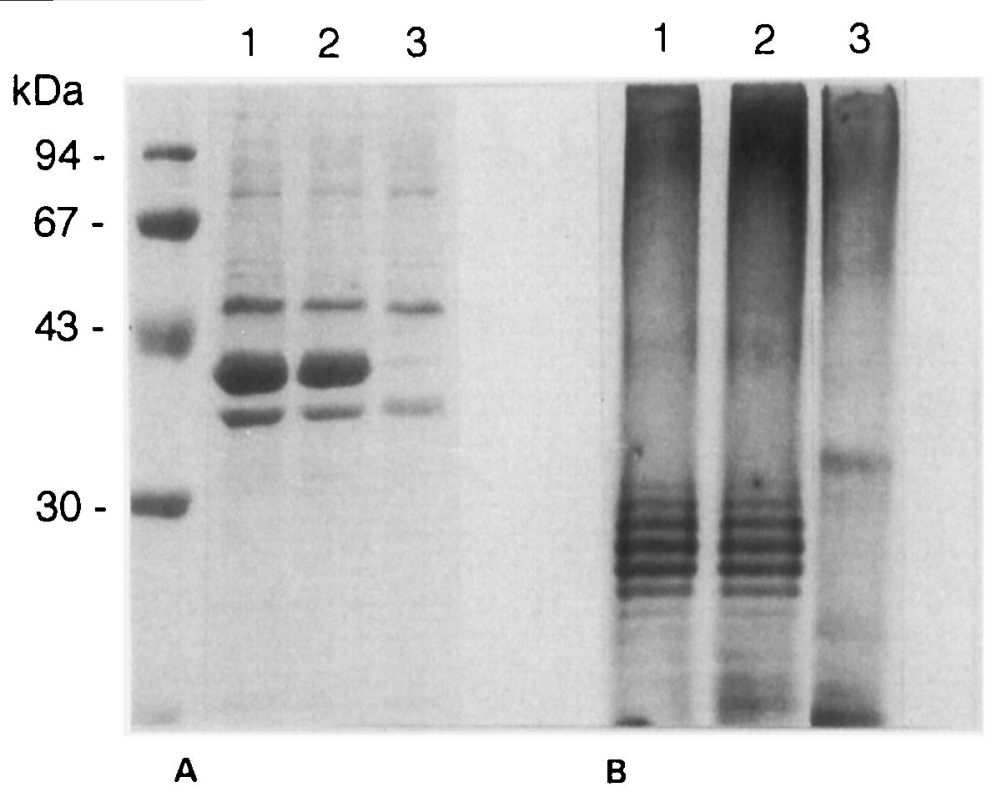

Fig. 3. A, SDS-PAGE of OMP preparations; B, silver-stained LPS profiles of the three E. aerogenes clinical isolates. Lane 1, isolate E1; 2, isolate E2; 3, isolate E3. Standard proteins of known molecular mass are shown on the left.

imipenem resistance. ${ }^{5}$ It has been reported that imipenem-resistant $E$. aerogenes mutants with altered OMP profiles and constitutive chromosomal $\beta$ lactamase production can be selected by other $\beta$ lactam agents such as cefotaxime, ${ }^{3}$ and an imipenemresistant clinical isolate of $E$. aerogenes was characterised by loss of a $40-\mathrm{kDa}$ OMP. ${ }^{4}$

The similarity of biotypes and ribotyping profiles of the isolates in the present study indicated that derivatives of a single bacterial strain that had undergone several mutations during exposure to antibiotics were being examined. The final imipenem-resistant mutant lacked, almost totally, a major OMP which, in its molecular mass, resembled the enterobacterial OmpF protein. Loss of this protein correlated, at least partly, with the significantly decreased rates of $\beta$-lactam penetration observed with this isolate. However, the simultaneous absence of the high molecular mass $\mathrm{O}$ antigenic LPS side-chains indicated an extensive alteration of the outer-membrane structure, which might, in turn, explain the successful transformation of isolate E3 with plasmid pNH5. Thus, although porin loss seems to be the final event leading to imipenem resistance in $E$. aerogenes, the contribution of other outer-membrane changes cannot be discounted.

According to the results presented here, stable derepression of the chromosomal cephalosporinase is indispensable for emergence of imipenem resistance. Cessation of class-I $\beta$-lactamase production, following introduction of plasmid $\mathrm{pNH} 5$ encoding $a m p D^{+}$, rendered isolate E3 fully susceptible to imipenem. It seems that the very slow inactivation of imipenem becomes enhanced by the presence of a potent outermembrane barrier, and that the two mechanisms act in a strongly synergic manner.

In a study conducted in 1988 by the Greek Society for Microbiology, ${ }^{23}$ high resistance rates for the common nosocomial pathogens were observed. Thus, among Enterobacter spp., 3\% were resistant to imipenem, and several policies, including restriction of imipenem usage, were initiated. In spite of this restriction policy, a second survey during 1992 found a resistance figure of $4 \% .^{24}$ If the hypothesis that cephalosporinase derepression is a pre-requisite for imipenem resistance is correct, the overprescription of some "popular" cephalosporins in Greek hospitals might share some responsibility for the maintenance and further development of resistance to imipenem.

We are grateful to Dr A. Vatopoulos for providing bacterial isolates and clinical data and Dr T. L. Pitt for helpful suggestions. We also thank Mrs D. Giannakoulia for technical assistance. This work was supported by the Greek Ministry of Health (Grant EO60).

\section{References}

1. Wiedemann B. Genetic and biochemical basis of resistance of Enterobacteriaceae to $\beta$-lactam antibiotics. $J$ Antimicrob Chemother 1986; 18 Suppl B: 31-38.

2. Yang $\mathrm{Y}$, Livermore DM, Williams RJ. Chromosomal $\beta$ lactamase expression and antibiotic resistance in Enterobacter cloacae. J Med Microbiol 1988; 25 : 227-233.

3. Hopkins JM, Towner KJ. Enhanced resistance to cefotaxime and imipenem associated with outer membrane protein alterations in Enterobacter aerogenes. $J$ Antimicrob Chemother 1990; 25 : 49-55.

4. Chow JW, Shlaes DM. Imipenem resistance associated with the loss of a $40 \mathrm{kDa}$ outer membrane protein in Enterobacter aerogenes. J Antimicrob Chemother 1991; 28: 499-504.

5. Tzouvelekis LS, Tzelepi E, Mentis AF, Vatopoulos AC, Tsakris A. Imipenem resistance in Enterobacter aerogenes is associated with derepression of chromosomal cephalosporinases and impaired permeability. FEMS Microbiol Lett 1992; 95: $195-200$

6. DeChamps C, Henquell C, Guelon D, Sirot D, Gazuy N, Sirot J. Clinical and bacteriological study of nosocomial infections due to Enterobacter aerogenes resistant to imipenem. J Clin Microbiol 1993; 31: 123-127. 
7. Honore N, Nicolas MH, Cole ST. Regulation of enterobacterial cephalosporinase production : the role of membrane-bound sensory transducer. Mol Microbiol 1989; 3: 1121-1130.

8. Sambrook J, Fritsch EF, Maniatis T. Molecular cloning: a laboratory manual, 2nd edn. Cold Spring Harbor, NY, Cold Spring Harbor Laboratory Press. 1989.

9. Garaizar J, Kaufmann ME, Pitt TL. Comparison of ribotyping with conventional methods for the type identification of Enterobacter cloacae. $J$ Clin Microbiol 1991; 29: 1303-1307.

10. Pitcher DG, Owen RJ, Dyal P, Beck A. Synthesis of a biotinylated DNA probe to detect ribosomal RNA cistrons in Providencia stuartii. FEMS Microbiol Lett 1987; 48 : 283-287.

11. National Committee for Clinical Laboratory Standards. Methods for dilution antimicrobial susceptibility tests for bacteria that grow aerobically. Approved Standard M7-A. Villanova, PA, USA, NCCLS. 1985.

12. Tzelepi E, Tzouvelekis LS, Vatopoulos AC, Mentis AF, Tsakris A, Legakis NJ. High prevalence of stably derepressed classI $\beta$-lactamase expression in multiresistant clinical isolates of Enterobacter cloacae from Greek hospitals. J Med Microbiol 1992; 37: 91-95.

13. Matthew M, Harris AM, Marshall, Ross GW. The use of analytical isoelectric focusing for detection and identification of $\beta$-lactamases. J Gen Microbiol $1975 ; 88$ : 169-178.

14. Filip C, Fletcher G, Wulff JL, Earhart CF. Solubilization of the cytoplasmic membrane of Escherichia coli by the ionic detergent sodium-lauryl sarcosinate. J Bacteriol 1973; 115: 717-722.

15. Hitchcock PJ, Brown TM. Morphological heterogeneity among Salmonella lipopolysaccharide chemotypes in silver-stained polyacrylamide gels. J Bacteriol 1983; 154: 269-277.
16. Nikaido $\mathrm{H}$, Rosenberg EY, Foulds J. Porin channels in Escherichia coli. studies with $\beta$-lactams in intact cells. $J$ Bacteriol 1983; 153: 232-240.

17. Büscher K-H, Cullmann W, Dick W, Opferkuch W. Imipenem resistance in Pseudomonas aeruginosa resulting from diminished expression of an outer membrane protein. Antimicrob Agents Chemother 1987; 31: 703-708.

18. Trias J, Nikaido $H$. Outer membrane protein D2 catalyzes facilitated diffusion of carbapenems and penems through the outer membrane of Pseudomonas aeruginosa. Antimicrob Agents Chemother 1990; 34: 52-57.

19. Livermore DM. Permeation of $\beta$-lactam antibiotics into Escherichia coli, Pseudomonas aeruginosa, and other Gramnegative bacteria. Rev Infect Dis 1988; 10: 691-698.

20. Nikaido $\mathbf{H}$. Outer membrane barrier as a mechanism of antimicrobial resistance. Antimicrob Agents Chemother 1989; 33: 1831-1836.

21. Lee EH, Nicolas MH, Kitzis MD, Pialoux G, Collatz E, Gutmann L. Association of two resistance mechanisms in a clinical isolate of Enterobacter cloacae with high-level resistance to imipenem. Antimicrob Agents Chemother 1991; 35: 1093-1098.

22. Raimondi A, Traverso A, Nikaido H. Imipenem- and meropenem-resistant mutants of Enterobacter cloacae and Proteus rettgeri lack porins. Antimicrob Agents Chemother 1991; 35: 1174-1180.

23. The Greek Society for Microbiology. Antibiotic resistance among gram-negative bacilli in 19 Greek hospitals. J Hosp Infect 1989; 14: 177-181.

24. Legakis NJ, Tzouvelekis LS, Tsakris A, Legakis JN, Vatopoulos AC. On the incidence of antibiotic resistance among aerobic gram-negative rods isolated in Greek hospitals. J Hosp Infect 1993; 24: 233-237. 